\title{
In Situ TEM Visualization on the Super Flexibility of Multi-layered Hydroxyapatite Nanobelts with Antibacterial Property
}

Mei-li Qi ${ }^{1,2}$, Shengkun Yao ${ }^{3,4}$, Zhennan Huang ${ }^{5}$, Yu-peng $\mathrm{Lu}^{2}$ and Reza Shahbazian-Yassar ${ }^{5}$

${ }^{1}$ School of Transportation Civil Engineering, Shandong Normal University, Ji'nan, Shandong, China (People's Republic), ${ }^{2}$ School of Materials Science and Engineering, Shandong University, Ji'nan, Shandong, China (People's Republic), ${ }^{3}$ School of Physics and Electronics, Shandong Normal University, Ji'nan, Shandong, China (People's Republic), ${ }^{4}$ Collaborative Innovation Center of Light Manipulations and Applications, Shandong Normal University, Ji'nan, Shandong, China (People's Republic), ${ }^{5}$ University of Illinois at Chicago, Chicago, Illinois, United States

*Corresponding author: rsyassar@uic.edu, biosdu@sdu.edu.cn

Hydroxyapatite $\left(\mathrm{Ca}_{10}\left(\mathrm{PO}_{4}\right)_{6}(\mathrm{OH})_{2}\right.$, HA) has been the most extensively used bone implant material for the reconstruction and substitution of hard tissues ${ }^{[1]}$. However, the success and long-term survival of the implants are dependent on the prevention of bacterial infection after implant placement. On the other hand, there is a growing interest in flexible HA nanostructures, since being brittle with a poor fatigue resistance is the major limitation to use HA-based biomaterials as load-bearing bioceramics.

Here, multi-layered HA nanobelts with silver doping (Ag-HA) was successfully synthesized by a hydrothermal homogeneous precipitation method. In vitro studies showed that the Ag-HA nanobelts had good antibacterial property as well as enhanced bioactivity. Most importantly, cyclic compressionbending behavior of Ag-HA nanobelts with multi-layered structures was observed via in situ TEM technique, showing the superior flexibility of the nanobelts. In situ TEM enables us to visualize the deformation behavior of an individual Ag-HA nanobelt under an external force in real time. As illustrated in Fig. ${ }^{[2]}$, the Ag-HA nanobelt was subjected to eight continuous compression-bending cycles without any morphological change. The nanobelt can return to its original state after each of the compressionbending trials, even with extreme bending angles $\left(180^{\circ}\right)$. No crack formation was found inside the structure. To the best of our knowledge, we for the first time observed the unique flexibility of an individual Ag-HA nanobelt. Multi-layered nanobelts can endure more stress than single-layered nanobelts during the deformation process, due to the fact that multi-layered structures can slide next to each other easily to avoid stress concentration. The compression-bending behavior in Ag-HA nanobelts is in a platelike manner wherein layer sliding occurs and releases the in-plane strains efficiently on the neutral surface, just like bending a stack of paper ${ }^{[3]}$. Such a multi-layered structure plays an important role in contributing to the superior flexibility of the Ag-HA nanobelts.

This work provides new insight into the design of HA nanobelts with antibacterial properties and opens new opportunities for their load-supporting applications. It is also instructive to reveal the mechanical behavior of other ceramic materials in nanoscale. ${ }^{[4]}$ 


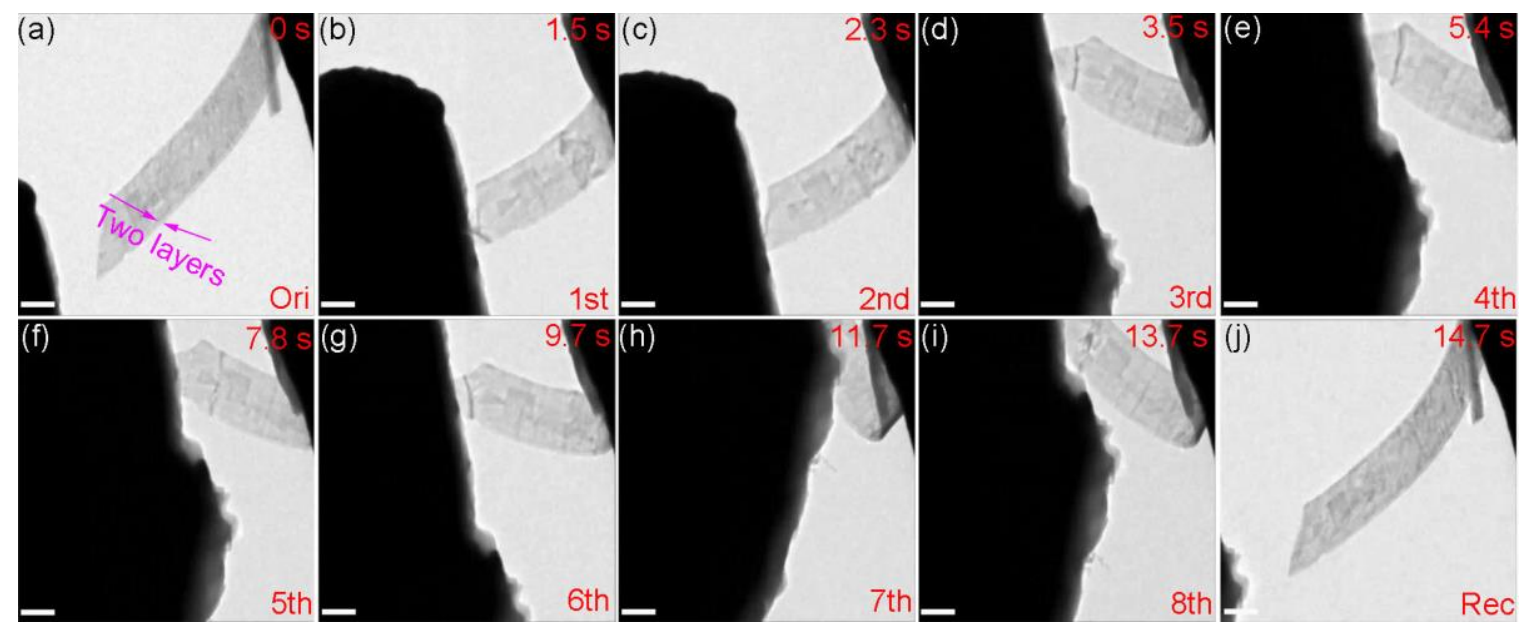

Figure 1. In situ TEM snapshot progress of an individual Ag-HA nanobelt in the eight compressionbending-recovery cycles. (a) an original nanobelt with two layers, (b-i) the nanobelt during the first to the eight bending cycles, (j) the nanobelt after recovery. Scale bars in the images are $200 \mathrm{~nm}[2]$.

\section{References}

[1] Wang Y, et al. Mater. Sci. Eng., C 108 (2020), p.110408.

[2] Qi M, et al. CrystEngComm 9 (2018), p.1304.

[3] Qi M, et al. CrystEngComm 8 (2018), p.1031.

[4] The authors acknowledge funding from the National Science Foundation of China (Award No. 11947117), Doctoral Scientific Research Foundation of Shandong Jiaotong University (Award No. 30550004919), and Postdoctoral Science Foundation of China (2019M660164). We also thank UIC Research Resources Center for assisting the usage of their equipment and instrumentation. 